\title{
Draft Genome Sequence and Transcriptional Analysis of Rosellinia necatrix Infected with a Virulent Mycovirus
}

\author{
Takeo Shimizu, Satoko Kanematsu, and Hajime Yaegashi†
}

First, second, and third authors: Division of Apple Research, Institute of Fruit Tree and Tea Science, National Agriculture and Food Research Organization (NARO), 92-24 Nabeyashiki, Shimokuriyagawa, Morioka, Iwate 020-0123, Japan.

Current address of first author: Division of Grape and Persimmon Research, Institute of Fruit Tree and Tea Science, NARO, 301-2 Mitsu, Akitsu, Hiroshima 739-2492.

Current address of second author: NARO Headquarter, 3-1-1 Kannondai, Tsukuba, Ibaraki 305-8517, Japan.

Accepted for publication 18 April 2018.

\begin{abstract}
Understanding the molecular mechanisms of pathogenesis is useful in developing effective control methods for fungal diseases. The white root rot fungus Rosellinia necatrix is a soilborne pathogen that causes serious economic losses in various crops, including fruit trees, worldwide. Here, using next-generation sequencing techniques, we first produced a $44-\mathrm{Mb}$ draft genome sequence of $R$. necatrix strain W97, an isolate from Japan, in which 12,444 protein-coding genes were predicted. To survey differentially expressed genes (DEGs) associated with the pathogenesis of the fungus, the hypovirulent W97 strain infected with Rosellinia necatrix megabirnavirus 1 (RnMBV1) was used for a comprehensive transcriptome

analysis. In total, 545 and 615 genes are up- and down-regulated, respectively, in $R$. necatrix infected with RnMBV1. Gene ontology and Kyoto Encyclopedia of Genes and Genomes pathway analyses of the DEGs suggested that primary and secondary metabolism would be greatly disturbed in $R$. necatrix infected with RnMBV1. The genes encoding transcriptional regulators, plant cell wall-degrading enzymes, and toxin production, such as cytochalasin E, were also found in the DEGs. The genetic resources provided in this study will accelerate the discovery of genes associated with pathogenesis and other biological characteristics of $R$. necatrix, thus contributing to disease control.
\end{abstract}

Rosellinia necatrix is a soilborne phytopathogenic fungus belonging to the order Xylariales that causes white root rot disease in a wide range of plant species, especially in perennial woody plants, including apple, pear, grape, orange, olive, avocado, and coffee throughout the world (Pliego et al. 2012; ten Hoopen and Krauss 2006). R. necatrix spreads in soil by mycelial growth, which proliferates and aggregates on the roots of host plants (Pliego et al. 2009). The aggregated mycelia penetrate into root tissues through natural opening sites, such as lenticels and wounds, or directly using penetrating sclerotia, and then invade the xylem. The infected plants gradually decline and finally die, and the disease spreads to the adjacent plants in the infested orchard.

Fungicides are conventionally used to control $R$. necatrix. The diseased roots are exposed by digging soil and drenching with fungicide. However, this method is laborious and unreliable because the pathogen can survive within the diseased roots and root debris for years, resulting in recurrent infections after a suppressive period. In addition, there are concerns regarding the unexpected negative effects of fungicides on ecosystems. Thus, it is necessary to develop more efficient and reliable methods than conventional ones to control this disease.

†Corresponding author: H. Yaegashi; E-mail: hyae@affrc.go.jp

Funding: This work was supported by JSPS KAKENHI grants 25292031 and 17K15232, and the Program for Promotion of Basic and Applied Researches for Innovation in Bio-Oriented Industries.

Accession number(s): This whole-genome shotgun project has been deposited in DDBJ/ENA/GenBank under BioProject number PRJDB3312 with GenBank accession numbers BBSO02000001 to BBSO02001209 for contigs and DF977446 to DF977684 for scaffolds. This is the second version of the draft genome sequence in the W97 strain of $R$. necatrix and replaces the first version number BBSO01000000.

*The $\boldsymbol{e}$-Xtra logo stands for "electronic extra" and indicates that five supplementary tables are published online.

(c) 2018 The American Phytopathological Society
Understanding the molecular mechanisms of pathogenesis is required to develop new control methods for $R$. necatrix. Previously, we investigated the contribution of melanin to the pathogenesis of $R$. necatrix (Shimizu et al. 2014), because melanin plays crucial roles in the pathogenesis of some fungi (Kubo and Furusawa 1991). However, the repressed expression of melanin biosynthetic genes revealed that it is dispensable for virulence but important for survival in $R$. necatrix (Shimizu et al. 2014). The molecular mechanism of $R$. necatrix's pathogenesis has not been clarified yet. Uncovering genome information will accelerate the discovery of genes associated with pathogenesis and other biological characteristics of $R$. necatrix.

Mycoviruses commonly exist in a variety of fungi (Xie and Jiang 2014). In phytopathogenic fungi, some mycoviruses reduce the virulence of their host fungi, and thus, mycoviruses may be utilized for the biological control of fungal diseases. In R. necatrix, we found a bipartite double-stranded RNA (dsRNA) mycovirus designated as Rosellinia necatrix megabirnavirus 1 (RnMBV1) (Chiba et al. 2009). RnMBV1 clearly reduces the mycelial growth of $R$. necatrix and its virulence on apple trees (Chiba et al. 2009). Moreover, RnMBV1 is equally distributed within $R$. necatrix colonies and is horizontally transmitted between colonies through hyphal fusion (Yaegashi et al. 2011). Thus, RnMBV1 is a good candidate for biocontrol agent of white root rot caused by $R$. necatrix. In addition, the hypovirulence of $R$. necatrix caused by RnMBV1 infection may be useful in investigating the pathogenesis of $R$. necatrix.

In some fungi, differentially expressed genes (DEGs) associated with mycovirus infections have been subjected to transcriptomic analyses to investigate fungal phenotypes including pathogenesis. A cDNA microarray analysis representing 2,200 genes was performed in the chestnut blight fungus Cryphonectria parasitica infected with a hypovirus CHV1-EP713 (Allen et al. 2003). In this fungus, $295 \mathrm{DEGs}$ after the hypovirus infection were involved in a variety of biological processes. In Fusarium graminearum associated with Fusarium head blight, a genome-wide expression analysis at two different time points using a 3 '-tiling microarray revealed 
that Fusarium graminearum virus 1 strain-DK21 infection affected the expression of genes related to transcription, translation, metabolism, and transport (Cho et al. 2012). In addition, an RNA sequencing (RNA-seq) analysis was performed using four $F$. graminearum strains individually infected with two virulent and two cryptic mycoviruses (Lee et al. 2014). In this analysis, only 12 DEGs were responsive to all four independent mycoviral infections, suggesting that mycoviruses may have distinct mechanisms for establishing and maintaining their infections in fungal cells. In Sclerotinia sclerotiorum, 150 DEGs were down-regulated in a cDNA library prepared from the Sclerotinia sclerotiorum debilitation-associated RNA virus infected strain ( $\mathrm{Li}$ et al. 2008). They were classified into various categories of biological functions. Thus, a comprehensive transcriptomic analysis of a fungus infected with a mycovirus is an effective strategy to discover the genes associated with fungal pathogenesis and/or virus infection through the establishment of a pool of DEGs.

Here, we reveal the draft genome sequence of the R. necatrix W97 strain, and DEGs are surveyed by comparing the data sets of the virus-free W97 strain with those of the W97 strain infected with RnMBV1. The characterization of the DEGs provides clues to understanding the effects of RnMBV1 infection on whole genome transcription in $R$. necatrix.

\section{MATERIALS AND METHODS}

Fungal strain and RNA extraction. $R$. necatrix strain W97 was isolated from the root of a Japanese pear in Imari, Saga, Japan (Ikeda et al. 2006). This strain is deposited in the National Institute of Agrobiological Sciences (NIAS)/GeneBank as MAFF625116, and hereafter referred to as W97. The W97 strain infected with RnMBV1 (Chiba et al. 2009) was used in this study and is, hereafter, referred to as W97 (RnMBV1).

For RNA extraction, both strains were cultivated on Difco potato dextrose agar (Beckton Dickinson, Sparks, MD) plates overlaid with cellulose membranes for 5 days in the dark at $25^{\circ} \mathrm{C}$. The collected mycelia with cellulose membranes were homogenized using liquid nitrogen and a Multibead shocker (Yasui Kikai, Osaka, Japan).

Total RNA was extracted from the homogenized mycelia using the RNeasy Plant Mini Kit (Qiagen GmbH, Hilden, Germany). After DNaseI treatment, RNA samples were purified and subjected to RNA-seq and real-time PCR analyses.

Genome analysis. Fragmented mycelia of W97 were cultured in potato dextrose broth for 2 days (Kanematsu et al. 2004) and subjected to phenol/chloroform DNA extraction. Two genome libraries (fragment library and 8-kb paired-end library) were sequenced using the Roche GS FLX+ system by Takara Bio (Ohtsu, Japan). In addition, HiSeq paired-end reads were obtained from the Illumina (San Diego, CA) HiSeq 2000 platform by Macrogen (Tokyo, Japan). The reads obtained were subjected to a hybrid assembly using GS De Novo Assembler 'Newbler' version 2.8. The generated contigs were subjected to scaffolding and gap filling using error-corrected PacBio long reads (82,067 Reads) by PacBio SMART Analysis (AHA scaffolding module) and PBJelly.

The RNA-seq of W97 was performed on the Illumina HiSeq2000 platform by Macrogen using an RNA sample extracted from the mycelia of the W97 strain cultivated for 5 days on potato dextrose agar in the dark. The raw RNA-seq reads from biological triplicates of W97 strain were trimmed, low quality reads filtered, and mapped to the scaffolds using Tophat v.2.1.6 (Trapnell et al. 2009). The mapped reads were assembled using cufflinks (Trapnell et al. 2010) and merged using cuffmerge. The gene prediction was performed using AUGUSTUS (Hoff and Stanke 2013) and CodingQuarry ver.1.3 (Testa et al. 2015) after training using the assembled transcripts. The predicted genes were combined into a consensus gene structure using EvidenceModeler (Haas et al. 2008).

RNA-seq for the transcriptomic analysis. To investigate the transcription of whole genes in the R. necatrix genome, an RNA-seq analysis was performed using three independent RNA samples in both W97 and W97 (RnMBV1). For both $R$. necatrix strains, nextgeneration sequencing was performed by Macrogen (Global Headquarters, Seoul, Korea) using the HiSeq 2000 system (Illumina) with 100 -bp paired-end reads. The raw reads from biological triplicates of W97 and W97 (RnMBV1) strains are available at DDBJ Sequence Read Archive (accession numbers DRR111062 to DRR111067).

A statistical analysis of the expression data of W97 and W97 (RnMBV1) was performed by the empirical analysis of DGE (EDGE) in CLC Genomics Workbench 10.0.0 (CLC Bio, Aarhus, Denmark). A heat map of genes statistically evaluated by EDGE $(1.5<$ fold change $<-1.5$ and $P$ value of $<0.05)$ was also constructed by CLC Genomics Workbench 10.0.0 (CLC bio). The DEGs were identified using the following conditions: $4<$ fold change $<-4$ and false discovery rate (FDR) $P$ value of $<0.05$.

Real-time PCR analysis. Total RNA samples from W97 and W97 (RnMBV1) were used for cDNA synthesis with Superscript_III Reverse transcription (Invitrogen, Life Technologies, Carlsbad, CA). The resulting cDNAs were used as templates for real-time PCR.

Real-time PCR was performed using the Power SYBR_ Green Master Mix (Applied Biosystems, Foster City, CA) and gene-specific primers (Supplementary Table S1). Three replicates of each were analyzed on a 7300 Real-Time PCR system (Applied Biosystems) using the Absolute Quantification (standard curve) assay and relative transcription level was calculated using the actin gene for normalization according to the instructions of "Real-Time PCR systems Chemistry Guide" and "User Bulletin 2" in Applied Biosystems.

Gene ontology (GO) analyses by Blast2GO. GO analyses were performed with Blast2GO 2.8 downloaded from the website https://www.blast2go.com/.

GO enrichment and Kyoto Encyclopedia of Genes and Genomes (KEGG) pathway analyses were performed by Blast2GO 2.8 using the up- or down-regulated DEGs.

Analysis of cytochalasin E. A mycelial segment $(6 \mathrm{~mm}$ in diameter) of each strain was cultured in $50 \mathrm{ml}$ of malt dextrose broth $(0.2 \%$ malt extract, $0.2 \%$ glucose, and $0.01 \%$ Bact peptone in distilled water) at $25^{\circ} \mathrm{C}$ in the dark. After 3 or 4 weeks, the cultured mycelial mat and broth were separated, and the dry weight of mycelia was measured. Broth was combined from three replicates, filtrated with a $0.2-\mu \mathrm{m}$ syringe filter (PTFE; Advantec, Tokyo, Japan), and diluted 10,000 times in methanol. Samples were stored at $-30^{\circ} \mathrm{C}$ prior to further analysis.

An ACQUITY UPLC ultra performance liquid chromatograph (Waters, Milford, MA) consisting of a binary pump, an auto-sampler $\left(10^{\circ} \mathrm{C}\right)$, and a thermostatic column compartment maintained at $40^{\circ} \mathrm{C}$ was used for analyses here. Five microliters of sample was injected and separation was performed on a reverse-phase column, Supleco C8 AscenticsR-Express, $2.1 \times 50 \mathrm{~mm}$, with $2.7-\mu \mathrm{m}$ particles (SigmaAldrich, St. Louis, MO). Mobile phase A was water (0.1\% ammonium acetate) and mobile phase $\mathrm{B}$ was acetonitrile ( $0.1 \%$ formic acid). The gradient was started from $70 \% \mathrm{~A}$ and $30 \% \mathrm{~B}$, and reached $100 \% \mathrm{~B}$ via a linear gradient over $10 \mathrm{~min}$ at a flow rate of $0.2 \mathrm{ml} / \mathrm{min}$. The effluent was monitored by UV detection at $280 \mathrm{~nm}$.

All column effluent was directed into an ACQUITY TQD tandem quadrupole mass spectrometer (Waters). Ionization optimization of standard cytochalasin E for the MS/MS analysis was performed by direct infusion. The ESI source was operated in the positive-ion mode at a capillary and cone voltage of $52 \mathrm{~V}$, with collision energy of $24 \mathrm{eV}$. Ion source and desolvation temperatures were maintained at $125^{\circ} \mathrm{C}$. The product ion spectra of cytochalasin $\mathrm{E}$ were obtained by selecting the protonated molecular ion $[\mathrm{M}+\mathrm{Na}]^{+}$for collision and the fragment ion was scanned at $518.3>474.3 \mathrm{~m} / \mathrm{z}$.

For the calibration, a standard curve was generated to confirm the linear relationship between the peak area and the concentration of cytochalasin $\mathrm{E}$ in the sample solution. Commercial cytochalasin $\mathrm{E}$ (Sigma-Aldrich) was diluted to different concentrations $(0.5,1.0$, 3.0, 7.0, and $10.0 \mathrm{nM}$ ) with methanol. Cytochalasin E analysis was undertaken by Nippon Steel \& Sumikin Eco-Tech Corporation. 


\section{RESULTS}

Genome information of $\boldsymbol{R}$. necatrix. A virus-free $R$. necatrix strain W97 shows stable mycelial growth on, and virulence to, host plants. A draft genome sequencing of the W97 strain was performed. A total of 2,910,554 reads containing 888,063,893 nucleotides, including 652,001 paired-end reads, in addition to 53,323,488 pairedend HiSeq reads containing 5,371,832,691 nucleotides were obtained. After hybrid assembly of the reads obtained, 239 scaffolds were constructed (N50: 549,874 bp) including 1,209 contigs with 44,257,831 nucleotides. RNA-seq data (total 209,640,910 raw reads) from biological triplicates of W97 strain were utilized for gene prediction. In the $44-\mathrm{Mb}$ draft genome sequence, 12,444 protein-coding genes were predicted, with 9,347 (75.1\%) of the sequences assigned predicted functions through a BLASTX algorithm-based analysis (E value $<10^{-3}$ ) against sequences in the NCBI nonredundant (nr) database and in InterProScan using Blast2GO. The species receiving the top hits were Eutypa lata and Pestalotiopsis fici, which, like $R$. necatrix, belong to the order Xylariales.

The annotated genes were subjected to GO analyses (Table 1). In biological process, the GO term metabolic process (GO:0008152)

TABLE 1. Gene ontology (GO) terms in biological process, molecular function, and cellular component represented by protein-coding genes in Rosellinia necatrix strain W97

\begin{tabular}{|c|c|c|}
\hline GO ID & GO term ${ }^{\mathrm{a}}$ & Sequence number \\
\hline \multicolumn{3}{|c|}{ Biological process } \\
\hline GO:0008152 & Metabolic process & 5139 \\
\hline GO:0009987 & Cellular process & 4067 \\
\hline GO:0044699 & Single-organism process & 2290 \\
\hline GO:0065007 & Biological regulation & 1241 \\
\hline GO:0051179 & Localization & 1231 \\
\hline GO:0071840 & $\begin{array}{l}\text { Cellular component } \\
\text { organization or biogenesis }\end{array}$ & 1135 \\
\hline GO:0050896 & Response to stimulus & 620 \\
\hline GO:0032502 & Developmental process & 251 \\
\hline GO:0023052 & Signaling & 202 \\
\hline GO:0000003 & Reproduction & 176 \\
\hline GO:0032501 & $\begin{array}{l}\text { Multicellular organismal } \\
\text { process }\end{array}$ & 141 \\
\hline GO:0051704 & Multi-organism process & 98 \\
\hline GO:0040007 & Growth & 49 \\
\hline GO:0040011 & Locomotion & 6 \\
\hline GO:0022610 & Biological adhesion & 3 \\
\hline GO:0048511 & Rhythmic process & 2 \\
\hline GO:0001906 & Cell killing & 1 \\
\hline \multicolumn{3}{|c|}{ Molecular function } \\
\hline GO:0003824 & Catalytic activity & 4260 \\
\hline GO:0005488 & Binding & 3954 \\
\hline GO:0005215 & Transporter activity & 437 \\
\hline GO:0001071 & $\begin{array}{l}\text { Nucleic acid binding } \\
\text { transcription factor activity }\end{array}$ & 257 \\
\hline GO:0005198 & Structural molecule activity & 195 \\
\hline GO:0030234 & Enzyme regulator activity & 112 \\
\hline GO:0000988 & $\begin{array}{l}\text { Protein binding transcription } \\
\text { factor activity }\end{array}$ & 51 \\
\hline GO:0016209 & Antioxidant activity & 33 \\
\hline GO:0060089 & Molecular transducer activity & 30 \\
\hline GO:0009055 & Electron carrier activity & 29 \\
\hline GO:0004872 & Receptor activity & 15 \\
\hline GO:0031386 & Protein tag & 7 \\
\hline GO:0045182 & Translation regulator activity & 4 \\
\hline GO:0045735 & Nutrient reservoir activity & 4 \\
\hline GO:0016530 & Metallochaperone activity & 3 \\
\hline \multicolumn{3}{|c|}{ Cellular component } \\
\hline GO:0005623 & Cell & 3345 \\
\hline GO:0043226 & Organelle & 2605 \\
\hline GO:0016020 & Membrane & 1399 \\
\hline GO:0032991 & Macromolecular complex & 1241 \\
\hline GO:0031974 & Membrane-enclosed lumen & 533 \\
\hline GO:0005576 & Extracellular region & 103 \\
\hline GO:0019012 & Virion & 43 \\
\hline GO:0009295 & Nucleoid & 18 \\
\hline
\end{tabular}

a The selected GOs belong to level 2 . contained the most genes. In molecular function, the GO term catalytic activity (GO:0003824) contained the most genes. Thus, W97 may produce a variety of metabolites. Furthermore, it was also revealed that a lot of genes were classified into the GOs related to regulation, such as biological regulation (GO:0065007) in biological process, nucleic acid binding transcription factor activity (GO:0001071), enzyme regulator activity (GO:0030234), protein binding transcription factor activity (GO:0000988), and translation regulator activity (GO:0045182) in molecular function.

Transcriptomic analysis using RNA-seq in $R$. necatrix. To characterize the transcriptional change caused by RnMBV1 infection in $R$. necatrix, transcriptomic analysis using RNA-seq was performed. The RNA-seq data including the raw reads from biological triplicates (W1: 66,260,128; W2: 76,890,282; and W3: $66,490,600)$ of W97 and those (M1: 63,042,264; M2: 67,530,378; and M3: $59,983,584)$ of W97 (RnMBV1) were subjected to statistical analysis to evaluate differential gene expression between W97 and W97 (RnMBV1). A total of 6045 genes were statistically evaluated, and a heat map of the genes showed similar expression patterns among W1, W2, and W3 or among M1, M2, and M3 (Fig. 1), supporting the reliability of our RNA-seq data.

To validate the RNA-seq data, 10 genes were selected based on the following conditions: $3<$ fold change $<-3$ and FDR $P$ value of $<0.05$. The real-time PCR analysis showed that these genes were regulated similarly to the expected transcriptional pattern from RNAseq data, except for cytochrome P450 (SAMD00023353_2500720) (Table 2). Notably, the fold change of the developmental regulator flbA gene (SAMD00023353_0102380) was 3.24 in RNA-seq data, but was 1.75 in real-time PCR data (Table 2 ). To identify more reliable DEGs, we adopted more stringent conditions $(4<$ fold change $<-4$ and FDR $P$ value of $<0.05)$ and identified 545 up- and 615 downregulated DEGs in this study.

GO analysis with Blast2GO using DEGs. The 545 up- and 615 down-regulated DEGs were analyzed for GO enrichment to statistically characterize them with GO terms. The results are summarized in Table 3. The GO terms common to both up- and downregulated genes were iron ion binding (GO:0005506), heme binding (GO:0020037), tetrapyrrole binding (GO:0046906), oxidoreductase activity, acting on paired donors, with incorporation or reduction of molecular oxygen (GO:0016705), and transition metal ion binding (GO:0046914). GO terms specific to the up-regulated genes were metal ion binding (GO:0046872), monooxygenase activity (GO:0004497), cation binding (GO:0043169), cellular carbohydrate catabolic process (GO:0044275), and ion binding (GO:0043167). GO

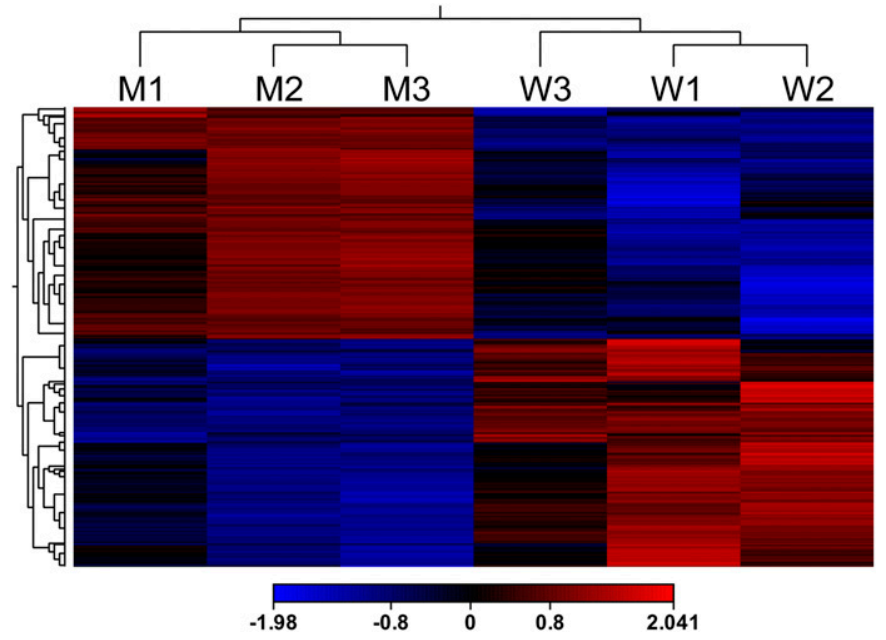

Fig. 1. Hierarchical clustering of genes differentially expressed between W97 and W97 (RnMBV1). A total of 6045 genes were extracted by empirical analysis of DGE among triplicates of W97 (W1, W2, and W3) and W97 (RnMBV1) (M1, M2, and M3). Red and blue indicate up- and down-regulation, respectively. 
terms specific to the down-regulated genes were oxidoreductase activity (GO:0016491), catalytic activity (GO:0003824), oxidationreduction process (GO:0055114), single-organism metabolic process (GO:0044710), and cofactor binding (GO:0048037). The GO terms selected by this analysis were related to oxidoreduction enzymes or cofactors for their functional activation in metabolism, suggesting that metabolic pathways may be greatly influenced in W97 (RnMBV1).

KEGG pathway analysis with Blast2GO using DEGs. To identify the metabolic pathways influenced by RnMBV1 infection in $R$. necatrix, a KEGG pathway analysis was performed using the DEGs (Table 4, Supplementary Tables S2 and S3).

For the up-regulated genes, 20 metabolic pathways that involved 28 genes were identified. For the down-regulated genes, 50 metabolic pathways that involved 88 genes were identified. Thus, many metabolic pathways, including primary and secondary metabolism, were affected by RnMBV1 infection in accordance with the results

TABLE 2. Genes selected for the validation of RNA-seq data

\begin{tabular}{|c|c|c|c|}
\hline \multirow[b]{2}{*}{ Gene ID } & \multirow[b]{2}{*}{ Putative function } & \multicolumn{2}{|c|}{ Fold change $^{\mathrm{a}}$} \\
\hline & & $\begin{array}{l}\text { RNA } \\
\text { seq }\end{array}$ & Real-time PCR \\
\hline $\begin{array}{l}\text { SAMD00023353 } \\
2300940\end{array}$ & $\begin{array}{l}\text { 2og-fe oxygenase } \\
\text { family protein }\end{array}$ & 11.47 & $35.34(4.52)$ \\
\hline $\begin{array}{l}\text { SAMD00023353 } \\
\quad 8300300\end{array}$ & $\begin{array}{l}\text { Cell division cycle } \\
\text { ATPase }\end{array}$ & 6.38 & $3.10(0.31)$ \\
\hline $\begin{array}{l}\text { SAMD00023353 } \\
\text { _0800540 }\end{array}$ & $\begin{array}{l}\text { AMP-binding \& Acyl- } \\
\text { protein }\end{array}$ & 4.40 & $7.40(1.04)$ \\
\hline $\begin{array}{l}\text { SAMMD00023353 } \\
\text { _1301510 }\end{array}$ & $\begin{array}{l}\text { Het domain-containing } \\
\text { protein }\end{array}$ & 3.45 & $3.08(0.34)$ \\
\hline $\begin{array}{l}\text { SAMMD00023353 } \\
\text { _0102380 }\end{array}$ & $\begin{array}{l}\text { Developmental } \\
\text { regulator flbA }\end{array}$ & 3.24 & $1.75(0.24)$ \\
\hline \multicolumn{4}{|l|}{ SĀMD00023353 } \\
\hline 7500260 & Phospholipase D p1 & -3.69 & $-10.72(1.78)$ \\
\hline $\begin{array}{l}\text { SAMD00023353 } \\
\text { _2200130 }\end{array}$ & $\begin{array}{l}\text { D-isomer specific 2- } \\
\text { hydroxyacid } \\
\text { dehydrogenase }\end{array}$ & -5.18 & $-4.54(1.12)$ \\
\hline \multicolumn{4}{|l|}{ SAMD00023353 } \\
\hline 2500720 & Cytochrome p450 & -6.72 & $4.83(0.51)$ \\
\hline $\begin{array}{l}\text { SAMMD00023353 } \\
\text { _1800920 }\end{array}$ & $\begin{array}{l}\text { Aspartyl-tRNA } \\
\text { synthetase }\end{array}$ & -7.34 & $-28.70(3.75)$ \\
\hline $\begin{array}{l}\text { SAMD00023353 } \\
\text { _1301810 }\end{array}$ & $\begin{array}{l}\text { Abundant perithecial } \\
\text { protein }\end{array}$ & -14.39 & $-3.81(0.46)$ \\
\hline
\end{tabular}

a The fold change values originated from RNA-seq data (evaluated using false discovery rate $P<0.05$ ) and from real-time PCR data (standard deviation).

TABLE 3. Gene ontology (GO) with up- and down-regulated genes

\begin{tabular}{ll}
\hline GO ID & \multicolumn{1}{c}{ GO term } \\
\hline Common & \\
GO:0005506 & $\begin{array}{l}\text { Iron ion binding } \\
\text { GO:0020037 }\end{array}$ \\
GO:0046906 & $\begin{array}{l}\text { Tetrapyrrole binding } \\
\text { Oxidoreductase activity, acting on paired } \\
\text { donors, with incorporation or reduction of } \\
\text { GO:0016705 }\end{array}$ \\
& molecular oxygen \\
Transition metal ion binding \\
GO:0046914 & Metal ion binding \\
Up & Monooxygenase activity \\
GO:0046872 & Cation binding \\
GO:0004497 & Cellular carbohydrate catabolic process \\
GO:0043169 & Ion binding \\
GO:0044275 & Oxidoreductase activity \\
GO:0043167 & Catalytic activity \\
Down & Oxidation-reduction process \\
GO:0016491 & Single-organism metabolic process \\
GO:0003824 & Cofactor binding \\
GO:0055114 & \\
GO:0044710 & \\
GO:0048037 &
\end{tabular}

of the GO enrichment analysis. Furthermore, the down-regulated genes were contained in more divergent metabolic pathways than the up-regulated genes, suggesting that metabolism may be generally inactivated in W97 (RnMBV1).

The cytochalasin biosynthetic gene cluster in $R$. necatrix. $R$. necatrix produces cytochalasin $\mathrm{E}$ as a secondary metabolite (Kanematsu et al. 1997). The cytochalasin biosynthetic gene cluster has been identified in Aspergillus clavatus NRRL1, which produces cytochalasin E and K (Qiao et al. 2011). Thus, the presence of the cytochalasin biosynthetic gene cluster was investigated in the genome of W97.

A putative cytochalasin biosynthetic gene cluster was identified in W97 (Table 5, Fig. 2A). Nine genes homologous to the cytochalasin biosynthetic gene cluster of A. clavatus NRRL1 (Fig. 2B) were located within a $\sim 36-\mathrm{kb}$ region, ranging from SAMD00023353 5500630 to SAMD00023353_5500720, of the $R$. necatrix genome. They encoded a nonribosomal peptide synthetase/polyketide synthase (SAMD00023353_5500630) for the production of the cytochalasin backbone structure, modification enzymes (SAMD00023353 5500640, SAMD00023353_5500660, SAMD00023353_5500670, SAMD00023353_5500680, SAMD00023353_5500690, SAMD00023353_5500700, and SAMD00023353_5500710), and a pathway-specific regulator (SAMD00023353_5500720). Interestingly, these genes, without the regulator gene, were down-regulated in W97 (RnMBV1). Real-time PCR analysis was conducted to verify down-regulation of six genes (SAMD00023353_5500630,_5500660, _5500670,_5500680,_5500700,_5500710; fold change <-4 and FDR $P$ value of $<0.05$ ) by RnMBV 1 infection. The results confirmed down-regulation of the six genes (Table 5). Quantitative analysis of cytochalasin $\mathrm{E}$ showed that amount of cytochalasin E production was significantly reduced in W97 (RnMBV1) compared with W97 after 3 -weeks cultivation period (Fig. 2C). This tendency was also confirmed in the analysis of 4-weeks cultures (data not shown).

\section{DISCUSSION}

Understanding the molecular mechanism of pathogenesis is important for developing effective control methods to protect various crops from $R$. necatrix. Next-generation sequencing techniques have been commonly utilized to obtain the genome sequence information of various fungi. This study first provided a draft genome sequence of $R$. necatrix strain W97, and its size and the number of the protein-coding genes were similar to those of major plant pathogenic fungi (Jung et al. 2008). Blast top hit species distribution reveals genes of $R$. necatrix are closely related to the other Xylariales fungi, a vascular pathogen, E. lata (Blanco-Ulate et al.

TABLE 4. The KEGG pathway analysis using up- and down-regulated genes

\begin{tabular}{lc}
\hline Category & Sequence number \\
\hline Up & \\
Amino acid metabolism & 2 \\
Biosynthesis of other secondary metabolites & 2 \\
Carbohydrate metabolism & 15 \\
Energy metabolism & 1 \\
Lipid metabolism & 5 \\
Translation & 1 \\
Others & 2 \\
Down & \\
Amino acid metabolism & 19 \\
Biosynthesis of other secondary metabolites & 7 \\
Carbohydrate metabolism & 16 \\
Energy metabolism & 7 \\
Lipid metabolism & 7 \\
Metabolism of cofactors and vitamins & 6 \\
Xenobiotics biodegradation and metabolism & 16 \\
Translation & 1 \\
Others & 9 \\
\hline
\end{tabular}

a The total number of genes in each category are shown. 
2013), and an endophytic fungus, P. fici (Wang et al. 2015). These results support the phylogenetic analysis of Xylariales, which is shown to be a monophyletic order containing seven families, including Xylariaceae (Rosellinia spp.), Amphisphaeriaceae (Pestalotiopsis spp.), and Diatypaceae (Eutypa spp.) (Smith et al. 2003). The draft genome of $R$. necatrix will be useful for comparing the molecular mechanisms in this root rot fungus with those in other closely related fungi with different lifestyles.

The characterization of DEGs is useful for understanding genes associated with fungal pathogenesis. In this study, a mycovirus, RnMBV1, which clearly reduces mycelial growth and the virulence of $R$. necatrix (Chiba et al. 2009), was used to survey DEGs associated with fungal pathogenesis. The statistical analysis identified 545 up- and 615 down-regulated DEGs in $R$. necatrix infected with RnMBV1. The present data are a resource of genetic information that can accelerate molecular investigations into the pathogenesis of R. necatrix.

There were 23 transcriptional regulators included in the DEGs (Supplementary Table S4). They consist of 19 up- and 4 downregulated genes. The transcriptional alteration of a large number of genes may be caused by a disordered regulatory network in the $R$. necatrix strain infected with RnMBV1. The factor(s) of RnMBV1 that are involved in the attenuation of $R$. necatrix pathogenesis are unknown. RnMBV1 mutants lacking RNA2 result in mild impacts on mycelial growth and virulence (Kanematsu et al. 2014), indicating that RNA2 of RnMBV1 is important for the attenuation of pathogenesis. Further functional analyses of two genes and/or other element(s) in RNA2 of RnMBV1 will be necessary to understand how RnMBV1 attenuates the pathogenesis of $R$. necatrix at the molecular level.

According to the GO enrichment analysis in which DEGs were used to identify significantly altered GOs in $R$. necatrix infected with RnMBV1, they contained genes related to primary and secondary metabolism. Disturbing the metabolism may cause the reduction in the virulence and growth of the $R$. necatrix strain infected with RnMBV1. In plant pathogenic fungi, one of metabolic pathways is plant cell wall degradation and plant cell wall-degrading enzymes play important roles not only in metabolism but also in pathogenicity. In the DEGs of the $R$. necatrix strain infected with RnMBV1, 7 up- and 10 down-regulated genes were found to encode enzymes related to plant cell wall degradation (Supplementary Table S5). They contain proteins, such as glucanase, cutinase, xylanase, and pectate lyase, suggesting that the inhibition of genes encoding plant cell wall-degrading enzymes may negatively impact $R$. necatrix's ability to invade host plants. The genes mentioned above will be a first target to investigate the molecular mechanism of pathogenesis in $R$. necatrix.

Additionally, fungal toxins play important roles in the pathogenesis of phytopathogenic fungi. Cytochalasin $\mathrm{E}$ is a toxic secondary metabolite produced by $R$. necatrix. Our present study revealed that a putative cytochalasin biosynthetic gene cluster was located in a 36-kb region of the $R$. necatrix genome. Although the genes contained in this gene cluster were highly homologous to those of A. clavatus NRRL1 (Qiao et al. 2011), the organization of the $R$. necatrix gene cluster was different (Fig. 2), suggesting that the gene organization of the clusters may have changed during the
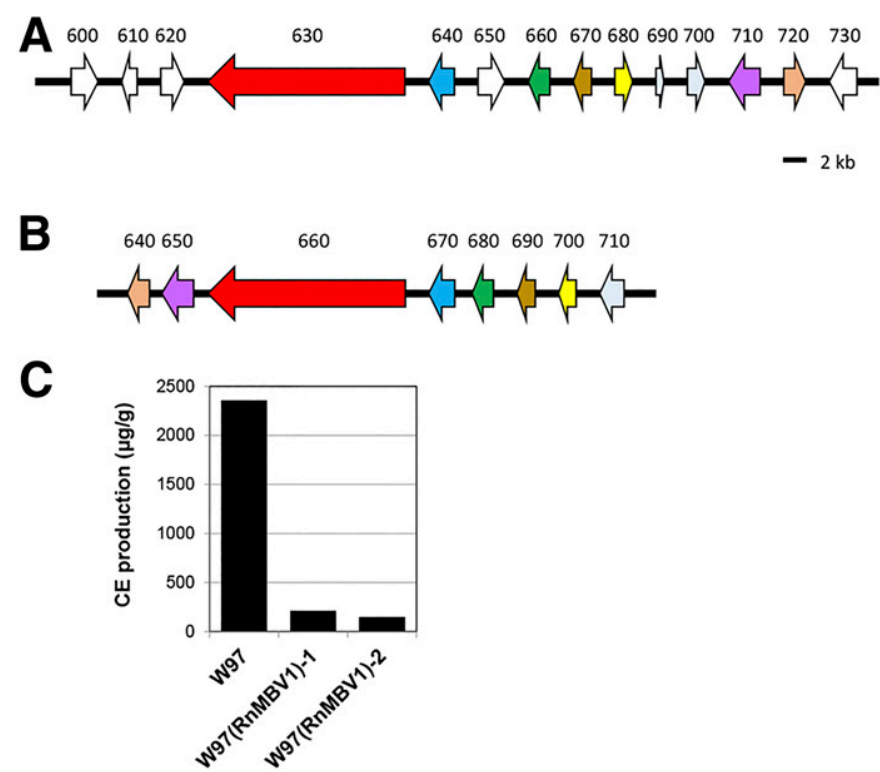

Fig. 2. Gene organization of the cytochalasin biosynthetic gene cluster. A, Putative cytochalasin biosynthetic gene cluster found in the Rosellinia necatrix genome. The numbers above the genes indicate the ' $\mathrm{xxx}$ ' part of the gene ID SAMD00023353_5500xxx. B, Cytochalasin biosynthetic gene cluster previously identified in Aspergillus clavatus NRRL1. The numbers above the genes indicate the ' $\mathrm{xxx}$ ' part of the gene ID ACLA 078xxx. In A and B, the directions, approximate sizes, and gene order are shown, although the intergenic regions are not represented to scale. The same gene color indicates the same gene in $\mathbf{A}$ and $\mathbf{B}$. The homologs of the white genes in $\mathbf{A}$ were not found in B. C, Quantitative analysis of cytochalasin E production of W97 and duplicates of W97 (RnMBV1). After 3 weeks of cultivation of each strain, the amount of cytochalasin $\mathrm{E}$ in the broth was quantified and normalized by the dry weight of mycelia.

TABLE 5. Genes within the region containing the putative cytochalasin biosynthetic gene cluster in the Rosellinia necatrix strain W97 genome

\begin{tabular}{|c|c|c|c|c|}
\hline \multirow[b]{2}{*}{ Gene ID } & \multirow[b]{2}{*}{ Putative function } & \multirow[b]{2}{*}{ Cytochalasin gene ${ }^{a}$} & \multicolumn{2}{|c|}{ Fold change ${ }^{b}$} \\
\hline & & & RNA seq & Real-time PCR \\
\hline SAMD00023353_5500600 & MFS multidrug transporter & No & 1.71 & - \\
\hline SAMD00023353_5500610 & Aflatoxin B1 aldehyde reductase & No & -2.45 & - \\
\hline SAMD00023353_5500620 & Aldo keto protein & No & $-\overline{2.00}$ & - \\
\hline SAMD00023353_5500630 & Hybrid NRPS PKS & ACLA 078660 & $-1 \overline{0.59}$ & $-8.91(1.57)$ \\
\hline SAMD00023353_5500640 & Cytochrome P450 oxidoreductase & ACLA 078670 & -2.35 & - \\
\hline SAMD00023353_5500650 & Major facilitator superfamily protein & No & $-\overline{1.25}$ & - \\
\hline SAMD00023353_5500660 & Alpha beta hydrolase & ACLA 078680 & $-\underline{7.68}$ & $-8.63(2.30)$ \\
\hline SAMD00023353_5500670 & Conserved hypothetical protein & ACLA 078690 & $-\overline{6.87}$ & $-9.21(3.73)$ \\
\hline SAMD00023353_5500680 & Zinc-binding dehydrogenase & ACLA 078700 & $-1 \overline{0.77}$ & $-9.50(2.97)$ \\
\hline SAMD00023353_5500690 & Cytochrome P450 & ACLA 078710 & $\overline{-3.18}$ & - \\
\hline SAMD00023353_5500700 & Cytochrome P450 & ACLA 078710 & $-\overline{7.46}$ & $-5.98(1.45)$ \\
\hline SAMD00023353_5500710 & Monooxygenase & ACLA 078650 & $-\overline{9.65}$ & $-9.57(1.74)$ \\
\hline SAMD00023353_5500720 & C6 finger domain-containing protein & ACLA 078640 & $\overline{1.40}$ & - \\
\hline SAMD00023353_5500730 & Hypothetical protein & No & -1.86 & - \\
\hline
\end{tabular}

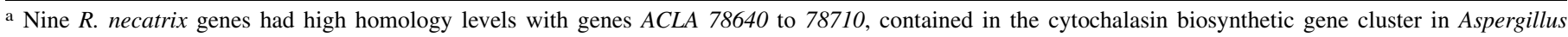
clavatus NRRL 1.

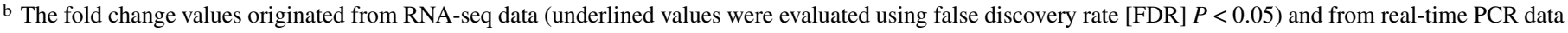
(standard deviation). In real-time PCR, the genes with fold change $<-4$ and FDR $P$ value of $<0.05$ were investigated. 
evolution of each fungi. However, the pathogenicity of $R$. necatrix was not dependent on the level of cytochalasin E (Kanematsu et al. 1997).

The DEGs identified after RnMBV1 infection in this study are candidate genes involved in the pathogenesis. In addition to the draft genome sequence and DEGs, genetic transformation methods are necessary to investigate molecular mechanisms of fungal pathogenesis. We previously established protoplast-polyethylene glycol and Agrobacterium-mediated transformation systems in R. necatrix (Kanematsu et al. 2004; Kano et al. 2011). Moreover, the application of autonomously replicating vectors to the transformation system could lead to increases in the transformation efficiency and the success of cotransformations with multiple vectors (Shimizu et al. 2012). These transformation systems are effective for the expression of an exogenous gene and the RNA silencing-mediated downregulation of exogenous and endogenous genes (Pliego et al. 2009; Shimizu et al. 2014, 2015; Yaegashi et al. 2013). By combining the genome sequence data with the genetic transformation methods in $R$. necatrix, further molecular studies of its pathogenesis will be greatly promoted, leading to the development of efficient and reliable methods of disease control.

\section{ACKNOWLEDGMENTS}

We thank H. Nakayashiki, Kobe University, for his technical assistance with the RNA-seq analyses; N. Oyama-Okubo, Institute of Vegetable and Floriculture Science, NARO, for supporting us in the use of UPLC-MS/MS; and L. Benyon, Ph.D., from Edanz Group for editing a draft of this manuscript.

\section{LITERATURE CITED}

Allen, T. D., Dawe, A. L., and Nuss, D. L. 2003. Use of cDNA microarrays to monitor transcriptional responses of the chestnut blight fungus Cryphonectria parasitica to infection by virulence-attenuating hypoviruses. Eukaryot. Cell 2:1253-1265.

Blanco-Ulate, B., Rolshausen, P. E., and Cantu, D. 2013. Draft genome sequence of the grapevine dieback fungus Eutypa lata UCR-EL1. Genome Announc. 1:e00228-e13.

Chiba, S., Salaipeth, L., Lin, Y. H., Sasaki, A., Kanematsu, S., and Suzuki, N. 2009. A novel bipartite double-stranded RNA mycovirus from the white root rot fungus Rosellinia necatrix: molecular and biological characterization, taxonomic considerations, and potential for biological control. J. Virol. 83:12801-12812.

Cho, W. K., Yu, J., Lee, K. M., Son, M., Min, K., Lee, Y. W., and Kim, K. H. 2012. Genome-wide expression profiling shows transcriptional reprogramming in Fusarium graminearum by Fusarium graminearum virus 1-DK21 infection. BMC Genomics 13:173.

Haas, B. J., Salzberg, S. L., Zhu, W., Pertea, M., Allen, J. E., Orvis, J., White, O., Buell, C. R., and Wortman, J. R. 2008. Automated eukaryotic gene structure annotation using evidence modeler and the program to assemble spliced alignments. Genome Biol. 9:R7.

Hoff, K. J., and Stanke, M. 2013. WebAUGUSTUS - a web service for training AUGUSTUS and predicting genes in eukaryotes. Nucleic Acids Res. 41(Web Server issue):W123-W128.

Ikeda, K., Nakamura, H., and Matsumoto, N. 2006. Comparison between Rosellinia necatrix isolates from soil and diseased roots in terms of hypovirulence. FEMS Microbiol. Ecol. 54:307-315.

Jung, K., Park, J., Choi, J., Park, B., Kim, S., Ahn, K., Choi, J., Choi, D., Kang, S., and Lee, Y. H. 2008. SNUGB: A versatile genome browser supporting comparative and functional fungal genomics. BMC Genomics 9:586.

Kanematsu, S., Arakawa, M., Oikawa, Y., Onoue, M., Osaki, H., Nakamura, H., Ikeda, K., Kuga-Uetake, Y., Nitta, H., Sasaki, A., Suzaki, K., Yoshida, K., and Matsumoto, N. 2004. A reovirus causes hypovirulence of Rosellinia necatrix. Phytopathology 94:561-568.
Kanematsu, S., Hayashi, T., and Kudo, A. 1997. Isolation of Rosellinia necatrix mutants with impaired cytochalasin E production and its pathogenicity. Jpn. J. Phytopathol. 63:425-431.

Kanematsu, S., Shimizu, T., Salaipeth, L., Yaegashi, H., Sasaki, A., Ito, T., and Suzuki, N. 2014. Genome rearrangement of a mycovirus Rosellinia necatrix megabirnavirus 1 affecting its ability to attenuate virulence of the host fungus. Virology 450-451:308-315.

Kano, S., Kurita, T., Kanematsu, S., and Morinaga, T. 2011. Agrobacterium tumefaciens-mediated transformation of the plant pathogenic fungus Rosellinia necatrix. Mikrobiologiia 80:86-92.

Kubo, Y., and Furusawa, I. 1991. Melanin biosynthesis: prerequisite for successful invasion of the plant host by appressoria of Colletotrichum and Pyricularia. Pages 205-218 in: The Fungal Spore and Disease Initiation in Plants and Animals. G. T. Cole and H. C. Hoch, eds. Plenum Publishing Corporation, New York.

Lee, K. M., Cho, W. K., Yu, J., Son, M., Choi, H., Min, K., Lee, Y. W., and Kim, K. H. 2014. A Comparison of transcriptional patterns and mycological phenotypes following infection of Fusarium graminearum by four mycoviruses. PLoS One 9:e100989.

Li, H., Fu, Y., Jiang, D., Li, G., Ghabrial, S. A., and Yi, X. 2008. Downregulation of Sclerotinia sclerotiorum gene expression in response to infection with Sclerotinia sclerotiorum debilitation-associated RNA virus. Virus Res. 135:95-106.

Pliego, C., Kanematsu, S., Ruano-Rosa, D., Vicente, A., Lopez-Herrera, C., Cazorla, F. M., and Ramos, C. 2009. GFP sheds light on the infection process of avocado roots by Rosellinia necatrix. Fungal Genet. Biol. 46:137-145.

Pliego, C., López-Herrera, C., Ramos, C., and Cazorla, F. M. 2012. Developing tools to unravel the biological secrets of Rosellinia necatrix, an emergent threat to woody crops. Mol. Plant Pathol. 13:226-239.

Qiao, K., Chooi, Y. H., and Tang, Y. 2011. Identification and engineering of the cytochalasin gene cluster from Aspergillus clavatus NRRL 1. Metab. Eng. 13:723-732.

Shimizu, T., Ito, T., and Kanematsu, S. 2012. Transient and multivariate system for transformation of a fungal plant pathogen, Rosellinia necatrix, using autonomously replicating vectors. Curr. Genet. 58:129-138.

Shimizu, T., Ito, T., and Kanematsu, S. 2014. Functional analysis of a melanin biosynthetic gene using RNAi-mediated gene silencing in Rosellinia necatrix. Fungal Biol. 118:413-421.

Shimizu, T., Yaegashi, H., Ito, T., and Kanematsu, S. 2015. Systemic RNA interference is not triggered by locally-induced RNA interference in a plant pathogenic fungus, Rosellinia necatrix. Fungal Genet. Biol. 76:27-35.

Smith, G. J. D., Liew, E. C. Y., and Hyde, K. D. 2003. The Xylariales: A monophyletic order containing 7 families. Fungal Divers. 13:185-218.

ten Hoopen, G. M., and Krauss, U. 2006. Biology and control of Rosellinia bunodes, Rosellinia necatrix and Rosellinia pepo. Crop Prot. 25:89-107.

Testa, A. C., Hane, J. K., Ellwood, S. R., and Oliver, R. P. 2015. CodingQuarry: Highly accurate hidden Markov model gene prediction in fungal genomes using RNA-seq transcripts. BMC Genomics 16:170.

Trapnell, C., Pachter, L., and Salzberg, S. L. 2009. TopHat: Discovering splice junctions with RNA-seq. Bioinformatics 25:1105-1111.

Trapnell, C., Williams, B. A., Pertea, G., Mortazavi, A., Kwan, G., van Baren, M. J., Salzberg, S. L., Wold, B. J., and Pachter, L. 2010. Transcript assembly and quantification by RNA-seq reveals unannotated transcripts and isoform switching during cell differentiation. Nat. Biotechnol. 28:511-515.

Wang, X., Zhang, X., Liu, L., Xiang, M., Wang, W., Sun, X., Che, Y., Guo, L., Liu, G., Guo, L., Wang, C., Yin, W.-B., Stadler, M., Zhang, X., and Liu, X. 2015. Genomic and transcriptomic analysis of the endophytic fungus Pestalotiopsis fici reveals its lifestyle and high potential for synthesis of natural products. BMC Genomics 16:28

Xie, J., and Jiang, D. 2014. New insights into mycoviruses and exploration for the biological control of crop fungal diseases. Annu. Rev. Phytopathol. 52: 45-68.

Yaegashi, H., Sawahata, T., Ito, T., and Kanematsu, S. 2011. A novel colonyprint immunoassay reveals differential patterns of distribution and horizontal transmission of four unrelated mycoviruses in Rosellinia necatrix. Virology 409:280-289

Yaegashi, H., Yoshikawa, N., Ito, T., and Kanematsu, S. 2013. A mycoreovirus suppresses RNA silencing in the white root rot fungus, Rosellinia necatrix. Virology 444:409-416. 